\title{
Cultural adaptation and translation of PEACH scale in Telugu language: Applicability in assessing auditory and communication skills of children with cochlear implant
}

\author{
S. B. Rathna Kumar', Vijay Raju Bollapalli², Udit Saxena ${ }^{3}$, Panchanan Mohanty ${ }^{4}$, Sakeena Shora ${ }^{5}$ \\ ${ }^{1}$ Ali Yavar Jung National Institute of Speech and Hearing Disabilities (Divyangjan), Mumbai, India, ${ }^{2}$ Composite Regional Centre for Empowerment \\ of Persons with Disabilities (Divyangjan), Davangere, India, ${ }^{3}$ MAA Institute of Speech and Hearing, Hyderabad, India, ${ }^{4}$ Department of English, GLA \\ University, Mathura, India, ${ }^{5}$ Spectrum Health Care, Mumbai, India
}

Purpose: The present study aimed to assess auditory and communication skills of children with cochlear implant $(\mathrm{Cl})$ as a function of the age at which they received $\mathrm{Cl}$.

Methods: Group I (Early Implanted Group: EIG) consisted of children ( $N=18)$ who received $\mathrm{Cl}$ in the age of 2 to 3 years $(\mathrm{M}=2.64)$. Group II (Later Implanted Group: LIG) consisted of children $(N=18)$ who received $\mathrm{Cl}$ in the age of 4 to 5 years $(M=4.64)$. The study constituted three phases; 1 ) adaptation and translation of the PEACH scale in Telugu language, 2) administration of Telugu PEACH scale on parents to assess auditory and communication skills of children with $\mathrm{Cl}$, and 3 ) scoring the responses of parents to obtain performance score of children in quiet and noisy communication environments.

Results: The results revealed no significant difference $(p>0.05)$ in the performance between EIG and LIG in both quiet and noisy communication environments. However, the children of both EIG and LIG demonstrated significantly lower $(p<0.05)$ performance in noisy communication environments when compared to performance in quiet communication environments.

Conclusions: From the findings of the study, it can be inferred that the age at which the children received $\mathrm{Cl}$ did not seem to be a major contributor in predicting the outcomes. Finally, the Telugu PEACH scale can be considered a clinically feasible tool in assessing the functional performance of children with $\mathrm{Cl}$.

Keywords: auditory and communication skills; early implanted group; later implanted group; quiet communication environments; noisy communication environments

\section{INTRODUCTION}

Hearing is an essential sense which plays a dynamic role in speech and language development, communication, and learning. However, children with congenital or early onset of hearing loss face challenges in developing and maintaining speech and language skills [1]. Hearing loss in children is a hidden disability, if undetected and untreated, it can lead to delay in speech and language development, social and emotional disturbances, and ultimate academic difficulties [2]. The disabling effects in children

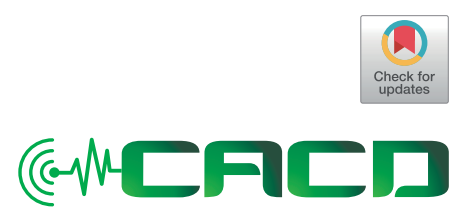

Received: August 8, 2020

Revision: December 25, 2020

Accepted: December 31, 2020

\section{Correspondence:}

S B Rathna Kumar

Ali Yavar Jung National Institute of Speech and Hearing Disabilities (Divyanjan), Bandra (west), Mumbai, India

Tel: $+91-8286157500$

Fax: +22-26404170

E-mail: sarathna@yahoo.co.in

(C) 2020 The Korean Association of SpeechLanguage Pathologists

This is an Open Access article distributed under the terms of the Creative Commons Attribution NonCommercial license (https://creativecommons.org/ licenses/by-nc/4.0/) which permits unrestricted noncommercial use, distribution, and reproduction in any medium, provided the original work is properly cited. 
arising from hearing loss can be minimized by detecting hearing loss as early as possible and providing early intervention through effective aural rehabilitative devices [2]. The selection of an appropriate rehabilitative device is a crucial component of an aural rehabilitation program. The currently available rehabilitative devices for children with hearing impairment are hearing aid (HA) and surgically implanted cochlear implant (CI). CI is presently one of the most considerable, successful, and advanced medical prostheses for individuals with hearing loss of severe-to-profound degree who receive limited benefit from conventional HAs [3].

The process of cochlear implantation provokes much anticipations and expectations in parents in terms of outcomes of their children with CI [4]. Hence, there is a need to monitor the outcomes of children at every phase of post-implant rehabilitation. The outcomes of children with CI can be assessed through objective and/or subjective measures. The objective measures provide information on the auditory and communicative performance of children within the highly structured acoustic environment under optimum listening conditions [5]. One of the subjective ways to evaluate auditory and communicative performance involves the administration of inventories and/or questionnaires to provide information on the functional performance of children based on observations of parents and/or caregivers of a child's behaviour in realworld communication environments [6]. In general, parents spend most of the time with their children in everyday communication environments. Hence, their reports based on observations are often more dependable and representative of the child's auditory related behaviour in a real-world communication environment when compared to objective assessments conducted in the controlled acoustic environments [7].

Several questionnaires and/or inventories have been developed for evaluating children's auditory related skills and the relative effectiveness of their hearing devices [8]. The necessity of including wider outcome measures, especially the parental reports while assessing the outcomes of children with CI has been emphasized [9-11]. Based on the high grades received in a critical review of subjective outcome evaluation questionnaires, the Parents' Evaluation of Aural/Oral Performance of Children (PEACH) scale [8] was found one of the more appropriate [5]. The PEACH scale focuses on auditory related behaviour of toddlers and older children with hearing loss ranging from mild to profound degree in terms of how they are hearing and communicating with others while using hearing devices in everyday communication environments
[8]. However, the outcomes of children with CI need to be assessed using subjective measures that are suitable for the population concerning their linguistic and cultural backgrounds [12]. Hence, the questionnaires and/or inventories developed for the English-speaking population may not be appropriate to the Indian population due to the high degree of diversity in their linguistic and cultural backgrounds.

Telugu is a Dravidian language spoken predominantly by people of Andhra Pradesh and Telangana states of south India. The 'Aarogyasri Healthcare Program' being implemented by the government of Andhra Pradesh and Telangana states bears the expenditure of the CI program including surgery and post-implant rehabilitation services for children placed below the poverty line. In addition to the state government healthcare scheme, the 'ADIP Scheme' being implemented by the government of India also offers a similar CI program for children falling below the poverty line. This has increased the number of children receiving CI in Andhra Pradesh and Telangana making them to have one of the largest CI populations in India [13]. The majority of children who have undergone and/or are undergoing cochlear implantation in Andhra Pradesh and Telangana states are native speakers of Telugu. Shortly, it is probable that the total number of children with CI who are native speakers of Telugu will not only continue to grow but will grow at an increasing rate. Hence, considering the increasing implications of subjective measures in assessing the functional outcomes of children with CI in everyday communication environments, and the growing number of children with CI in Andhra Pradesh and Telangana who are native speakers of Telugu, there is a need to adapt and translate PEACH scale for use in the Telugu language.

Age of implantation which is the age at which a child receives CI seems to be one of the most important variables to predict speech and language outcomes. Although, several studies have reported that children who receive $\mathrm{CI}$ at a very younger age are expected to achieve greater speech and language skills than children who receive $\mathrm{CI}$ at a later age $[10,14$ 17], some studies have reported otherwise [18-21]. Therefore, still there is nonalignment on whether children who receive $\mathrm{CI}$ at an earlier age only have greater potential in achieving higher linguistic competence than children who receive CI at a later age. Hence, the present study aimed to assess and compare the auditory and communication skills of children with $\mathrm{CI}$ as a function of the age at which they received CI using culturally adapted and translated PEACH scale in Telugu language to investigate whether early age of implantation is 
the main predictor of outcomes of children or whether factors such as pre-implant HA usage, duration of post-implant CI usage, type of post-implant intervention program and mode of communication at educational settings would add advantages for children who received $\mathrm{CI}$ in the later age.

\section{METHODS}

Present study constituted three phases. Phase I: adaptation and translation of PEACH scale for use in Telugu language; Phase II: administration of Telugu PEACH scale on parents of children with cochlear implant; Phase III: scoring the responses of parents to obtain PEACH scores for quiet subscale, noise subscale, and combined subscales.

\section{Phase I: Adaptation and Translation of PEACH scale for use in Telugu language}

PEACH scale [8] was adapted and translated for use in Telugu language to fulfil the aim of present study. An official permission has been obtained from the concerned author to adapt and translate the original scale for use in Telugu language for the present study. This scale consists of 13 items including two general items, six items related to listening and communicating in relatively quiet situations, four items related to listening and communicating in noisy situations, and one item on awareness and recognition of environmental sounds. A competent person who is a native speaker of Telugu and well versed in English as well reviewed the existing 13 items of the original PEACH scale. Few minor modifications to the content of the items $1,2,3,7,10,11$, and 12 were done to ensure the linguistic and cultural appropriateness of the concepts. The items 4, 5, 6, 8, 9, 13 were found to be linguistically and culturally appropriate and retained in their original forms. Also, one item related to listening and communicating in noisy situations was newly included in the scale and formed item 14 . The modified version of the PEACH scale was then reviewed by three experienced audiologists to ensure that the content of the items was familiar, easy to understand, and culturally appropriate. After necessary corrections made to the modified version as suggested by the reviewers, a different person who is well versed in English proofread the content of the items in the modified version of the PEACH scale.

A 'back-to-back' approach was used to translate the PEACH scale into Telugu language. The modified version of the PEACH scale was translated into Telugu by the same person who made modifications to the original version. During the process of translation, care was taken on syntactic structure, semanticity, familiarity, and ambiguity to maintain the original meaning of the content of the items. The translated version of the PEACH scale was then reviewed by three different experienced audiologists who are native speakers of Telugu and necessary corrections were made to the Telugu version as suggested by the reviewers. After this, the Telugu version was translated back to English by a different person who is well versed in both languages. To ensure the accuracy of the translation, a different person who is well versed in both languages reviewed the translated version and made necessary alterations to the content of the Telugu version so that the translated version was closest to the original version. Finally, the Telugu version was reviewed by six parents of children using hearing devices to ensure that the content was easy to understand and culturally appropriate.

The final version of the Telugu PEACH scale consisted of 14 items which include two general items (one item related to device usage, one item related to loudness discomfort), six items related to listening and communicating in quiet situations (responds to his/her name in a quiet situation, follows simple verbal instructions in a quiet situation, follows stories/ programs/serials on television in quiet situation, initiates/ participates in conversation in a quiet situation, recognizes familiar peoples' voices in a quiet situation, participates in conversation on phone in quiet situation), five items related to listening and communicating in noisy situations (responds to his/her name in a noisy situation, follows simple verbal instructions in a noisy situation, initiates/participates in conversations in noisy situations, participates in conversations in auto/car/bus/train, participates in conversation on phone in noisy situation) and one item related to awareness and recognition of environmental sounds other than voices.

\section{Phase II: Administration of Telugu PEACH scale on parents of children with cochlear implant}

Telugu PEACH scale was administered on the parents for assessing the auditory and communication skills of children with CI in quiet and noisy communication environments based on parent's observations of their child's behaviour in everyday communication environments.

\section{Participants}

The parents $(N=36)$ who are primary caregivers of children with CI served as participants in the present study. The age of parents was in the age range of 30 to 39 years $(M=33.22, S D=$ 
\pm 2.37 ). The parents have minimum educational qualification of higher secondary education, and the number of years of education was in the range of 12 to 17 years $(M=14.50, S D=$ $\pm 0.76)$. The age of children with CI was in the range of 8 to 11 years $(M=9.53, S D= \pm 0.25)$. The children were equally divided into two groups. Group I (Earlier Implanted Group: EIG) consisted of children $(N=18)$ in the age range of 8 to 10 years $(M=9.1, S D= \pm 0.24)$. The age at which these children received CI was in the age range of 2 to 3 years $(M=2.64, S D= \pm 0.16)$. The duration of CI usage, i.e., implant age was in the range of 6 to 7 years $(M=6.42, S D= \pm 0.18)$. The duration of pre-implant HA usage was in the range of 1 to 1.5 years $(M=1.24, S D=$ \pm 0.13 ). Five of EIG were using bimodal stimulation (i.e., CI in one ear and HA in the opposite ear) and remaining were limited to monaural CI.

Group II (Later Implanted Group, LIG) consisted of children $(N=18)$ in the age range of 9 to 11 years $(M=9.95, S D=$ $\pm 0.25)$. The age at which these children received CI was in the range of 4 to 5 years $(M=4.64, S D= \pm 0.21)$. The duration of $C I$ usage, i.e., implant age was in the range of 5 to 6 years $(M=$ $5.30, S D= \pm 0.18$ ). The duration of pre-implant HA usage was in the range of 2 to 3 years $(M=2.64, S D= \pm 0.16)$. Four of LIG were using bimodal stimulation (i.e., CI in one ear and HA in the opposite ear) and remaining were limited to monaural CI. All these children have attended 4 to 5 years of the post-implant intervention program in an oral mode of communication and currently attending age-appropriate mainstream education with oral language as a mode of communication. None of the children had additional disabilities or associated problems. The demographic, audiological, cochlear implant and speech processor details of children of EIG and LIG are shown in Tables 1 and 2.

\section{Procedure}

Telugu PEACH scale was administered on the parents with similar guidelines described by Ching \& Hill [8]. An interview session was arranged with parents to explain the items listed in the questionnaire and clarify the doubts of parents (if any) with the questionnaire. They were given a period of two weeks to observe the auditory and communication behaviour of their children concerning each question in everyday communication environments. They were instructed to record responses with as many examples as possible for each item in the questionnaire. After parents had completed the scale, an-

Table 1. Demographic, Audiological, Cochlear Implant and Speech Processor Information of Participants of EIG (N=18)

\begin{tabular}{|c|c|c|c|c|c|c|c|c|c|}
\hline Participant & Gender & CA & $\mathrm{Al}$ & IA & PI-HA & Implant & SP & SPS & MS \\
\hline EIG-1 & Female & $8 y 11 \mathrm{~m}$ & $2 y 6 m$ & 6 y $5 \mathrm{~m}$ & 1 y $1 \mathrm{~m}$ & CI24RECA & CP810 & ACE & Monaura \\
\hline EIG-2 & Female & 8 y 10 m & 2 y $6 m$ & 6 y 4 m & 1 y $2 \mathrm{~m}$ & Cl24RECA & CP910 & ACE & Bimodal \\
\hline EIG-3 & Female & 9 y $7 \mathrm{~m}$ & 2 y $10 \mathrm{~m}$ & 6 y $9 \mathrm{~m}$ & 1 y $5 \mathrm{~m}$ & Cl24RECA & Freedom & ACE & Monaura \\
\hline EIG-4 & Male & 8 y $11 \mathrm{~m}$ & 2 y $6 \mathrm{~m}$ & 6 y 5 m & 1 y $3 \mathrm{~m}$ & Cl24RECA & CP810 & ACE & Monaura \\
\hline EIG-5 & Male & 9 y $3 \mathrm{~m}$ & 2 y $8 \mathrm{~m}$ & $6 y 7 m$ & 1 y $2 \mathrm{~m}$ & CI24RECA & CP910 & ACE & Bimodal \\
\hline EIG-6 & Female & 9 y $4 \mathrm{~m}$ & 2 y $7 \mathrm{~m}$ & 6 y $9 m$ & 1 y $1 \mathrm{~m}$ & Cl24RECA & CP810 & ACE & Bimodal \\
\hline EIG-7 & Male & 9 y $5 \mathrm{~m}$ & 2 y $8 m$ & 6 y 2 m & 1 y $5 \mathrm{~m}$ & Cl24RECA & CP910 & ACE & Monaura \\
\hline EIG-8 & Female & 9 y $2 m$ & 2 y $9 \mathrm{~m}$ & 6 y $5 \mathrm{~m}$ & 1 y $2 \mathrm{~m}$ & CI24RECA & CP810 & ACE & Monaura \\
\hline EIG-9 & Female & 9 y $5 \mathrm{~m}$ & 2 y $11 \mathrm{~m}$ & 6 y 6 m & 1 y $6 \mathrm{~m}$ & Cl24RECA & CP910 & ACE & Monaura \\
\hline EIG-10 & Male & 9 y $3 \mathrm{~m}$ & 2 y $11 \mathrm{~m}$ & 6 y 4 m & 1 y $4 \mathrm{~m}$ & Cl24RECA & Freedom & ACE & Monaura \\
\hline EIG-11 & Female & 9 y $1 \mathrm{~m}$ & 2 y $10 \mathrm{~m}$ & 6 y 3 m & 1 y $5 \mathrm{~m}$ & Cl24RECA & CP910 & ACE & Bimodal \\
\hline EIG-12 & Female & 8 y 11 m & 2 y $9 m$ & 6 y 2 m & 1 y $3 \mathrm{~m}$ & Cl24RECA & Freedom & ACE & Monaura \\
\hline EIG-13 & Male & 9 y $3 \mathrm{~m}$ & 2 y $8 m$ & 6 y 7 m & 1 y $2 \mathrm{~m}$ & Cl24RECA & CP910 & ACE & Monaura \\
\hline EIG-14 & Female & 8 y 11 m & 2 y $6 \mathrm{~m}$ & 6 y 5 m & 1 y $2 \mathrm{~m}$ & Cl24RECA & CP810 & ACE & Monaura \\
\hline EIG-15 & Male & 8 y $9 m$ & 2 y $6 \mathrm{~m}$ & 6 y 3 m & 1 y $1 \mathrm{~m}$ & Cl24RECA & Freedom & ACE & Bimodal \\
\hline EIG-16 & Male & 8 y 11 m & $2 y 7 m$ & 6 y 4 m & 1 y $4 \mathrm{~m}$ & Cl24RECA & Freedom & ACE & Monaura \\
\hline EIG-17 & Female & 8 y 11 m & 2 y $4 \mathrm{~m}$ & 6 y 7 m & 1 y $1 \mathrm{~m}$ & CI24RECA & CP910 & ACE & Monaura \\
\hline EIG-18 & Female & $8 y 11 \mathrm{~m}$ & $2 y 7 m$ & 6 y $4 \mathrm{~m}$ & 1 y $3 \mathrm{~m}$ & CI24RECA & Freedom & ACE & Monaura \\
\hline
\end{tabular}

CA: Chronological Age; Al: Age of Implantation; IA: Implant Age; PI-HA: Pre-Implant Hearing Aid Usage; SP: Speech Processor; SPS: Speech Processing Strategy; MS: Mode of Stimulation; ACE: Advanced Combination Encoder. 
other structured interview session was arranged with parents to review the responses given by the parents. After reviewing the responses with them in this interview session, the authors further asked questions to parents for clarification of unclear examples reported by them. When the authors were not satisfied even after clarification with parents or if parents felt that they were still doubtful on some of the questions and responses, they were further allowed to clarify their doubts. After providing clarification to parents' doubts, they were further given another week to observe the auditory and communication behaviour of their children and record them with as many examples as possible. The clarification helped to increase the accuracy and number of response behaviours. The scale was administered twice on eight parents $(\mathrm{EIG}=4$ and $\mathrm{LIG}=4$ ) to check test-to-retest reliability of the Telugu PEACH scale.

Phase III: Scoring the responses of parents to obtain PEACH score and analysis of obtained data

\section{Scoring the responses}

The authors scored each item on the basis of responses given by parents on a five-point rating scale in the range between 0 and 4 as described by Ching \& Hill [8]. The ratings were never, seldom, sometimes, often and always, and assigned scores of $0,1,2,3$, and 4 respectively. An item was assigned a score of zero if no examples were provided by parents or if the child did not demonstrate any auditory and communicative response. A score of 1 was assigned if one or two examples were provided or if the parents reported that the child demonstrated auditory and communicative behaviour for $25 \%$ of the time. A score of 2 was assigned if three or four examples were provided or if the parents reported that the child demonstrated auditory and communicative behaviour for $50 \%$ of the time. A score of 3 was assigned if five or six examples were provided or if the parents reported that the child demonstrated auditory and communicative behaviour for $75 \%$ of the time. A maximum score of 4 was assigned if more than six examples were provided or if the parents reported that the child demonstrated the auditory and communicative behaviour for more than $75 \%$ of the time. The scores obtained for items $3,4,7,8$, 11 , and 12 were combined to derive performance score for communication in a quiet environment (PEACH score for quiet subscale) and the scores obtained for items 5, 6, 9, 10, 13, and 14 were combined to derive performance score for com-

Table 2. Demographic, Audiological, Cochlear Implant and Speech Processor Information of Participants of LIG (N=18)

\begin{tabular}{|c|c|c|c|c|c|c|c|c|c|}
\hline Participant & Gender & $\mathrm{CA}$ & $\mathrm{Al}$ & IA & PI-HA & Implant & SP & SPS & MS \\
\hline LIG-1 & Male & 9 y $8 \mathrm{~m}$ & 4 y 5 m & 5 y 3 m & $2 y 7 \mathrm{~m}$ & Cl24RECA & Freedom & ACE & Monaural \\
\hline LIG-2 & Female & 9 y 10 m & 4 y 3 m & 5 y $7 \mathrm{~m}$ & $2 y 1 \mathrm{~m}$ & Cl24RECA & CP910 & ACE & Monaural \\
\hline LIG-3 & Female & 10 y 1 m & 4 y 4 m & 5 y $9 \mathrm{~m}$ & $2 y 2$ m & CI24RECA & CP810 & ACE & Monaural \\
\hline LIG-4 & Male & 9 y $10 \mathrm{~m}$ & 4 y 6 m & 5 y $4 \mathrm{~m}$ & $2 y 8 \mathrm{~m}$ & Cl24RECA & CP910 & ACE & Monaural \\
\hline LIG-5 & Female & 10 y $1 \mathrm{~m}$ & 4 y $8 \mathrm{~m}$ & 5 y $5 \mathrm{~m}$ & $3 y 1 \mathrm{~m}$ & CI24RECA & CP810 & ACE & Monaural \\
\hline LIG-6 & Male & 9 y 10 m & 4 y 5 m & 5 y $5 \mathrm{~m}$ & $2 y 9 \mathrm{~m}$ & CI24RECA & CP910 & ACE & Monaural \\
\hline LIG-7 & Male & 10 y 1 m & 4 y 8 m & 5 y $5 \mathrm{~m}$ & 3 y 1 m & CI24RECA & CP810 & ACE & Monaural \\
\hline LIG-8 & Female & 9 y $11 \mathrm{~m}$ & 4 y 7 m & 5 y 4 m & 3 y $1 \mathrm{~m}$ & CI24RECA & CP810 & ACE & Bimodal \\
\hline LIG-9 & Female & 9 y 11 m & 4 y 9 m & 5 y 2 m & 3 y 1 m & Cl24RECA & CP810 & ACE & Monaural \\
\hline LIG-10 & Female & 9 y $8 m$ & 4 y 3 m & 5 y $5 \mathrm{~m}$ & $2 y 2 \mathrm{~m}$ & Cl24RECA & Freedom & ACE & Monaural \\
\hline LIG-11 & Female & 9 y $11 \mathrm{~m}$ & 4 y 10 m & 5 y $1 \mathrm{~m}$ & 3 y $1 \mathrm{~m}$ & Cl24RECA & Freedom & ACE & Bimodal \\
\hline LIG-12 & Male & 10 y 2 m & 4 y 9 m & 5 y $5 \mathrm{~m}$ & 3 y $1 \mathrm{~m}$ & Cl24RECA & CP910 & ACE & Monaural \\
\hline LIG-13 & Male & 9 y $2 \mathrm{~m}$ & 4 y 7 m & 5 y 2 m & 2y $4 \mathrm{~m}$ & Cl24RECA & CP810 & ACE & Bimodal \\
\hline LIG-14 & Female & 9 y $11 \mathrm{~m}$ & 4 y 9 m & 5 y $2 \mathrm{~m}$ & $2 y 9 \mathrm{~m}$ & Cl24RECA & CP810 & ACE & Monaural \\
\hline LIG-15 & Male & 10 y 2 m & 4 y 8 m & 5 y $6 \mathrm{~m}$ & 3 y 1 m & Cl24RECA & CP910 & ACE & Monaural \\
\hline LIG-16 & Female & 10 y 3 m & 4 y 11 m & 5 y 4 m & 2 y $5 \mathrm{~m}$ & Cl24RECA & Freedom & ACE & Monaural \\
\hline LIG-17 & Male & 9 y $11 \mathrm{~m}$ & 4 y 10 m & 5 y $1 \mathrm{~m}$ & 2 y 4 m & Cl24RECA & CP910 & ACE & Bimodal \\
\hline LIG-18 & Female & 10 y $1 \mathrm{~m}$ & 4 y $11 \mathrm{~m}$ & 5 y $2 \mathrm{~m}$ & 2 y $9 m$ & CI24RECA & Freedom & ACE & Monaural \\
\hline
\end{tabular}

CA: Chronological Age; Al: Age of Implantation; IA: Implant Age; PI-HA: Pre-Implant Hearing Aid Usage; SP: Speech Processor; SPS: Speech Processing Strategy; MS: Mode of Stimulation; ACE: Advanced Combination Encoder. 
munication in a noisy environment (PEACH score for noise subscale) for each child of EIG and LIG. Finally, the two subscale scores were summed to derive an overall performance score (overall PEACH score). The obtained scores of each subject were then converted into percentage PEACH scores (\%).

\section{Analysis of data}

Mean PEACH scores (\%) obtained by participants of each group, i.e., EIG, LIG, and combined group (CG) for each subscale, i.e., quiet subscale, noise subscale, and overall score were calculated. The mean PEACH scores (\%) were subjected to independent sample t-test in order to find out significant difference in the auditory and communication skills between participants of EIG and LIG for each subscale, i.e., quiet sub- scale, noise subscale, and overall score separately. The data were further subjected to one-way ANOVA in order to find out significant difference in the auditory and communicative performance of participants of each group, i.e., EIG, LIG and CG separately among quiet subscale score, noise subscale score, and overall score.

\section{RESULTS}

The results of Pearson's correlation coefficient revealed no significant difference in PEACH scores for quiet subscale, noise subscale, and combined subscale (overall score) when the Telugu PEACH scale was re-administered on a total of eight parents (EIG $=4$ and $L I G=4)$ showing a high test-to-re-

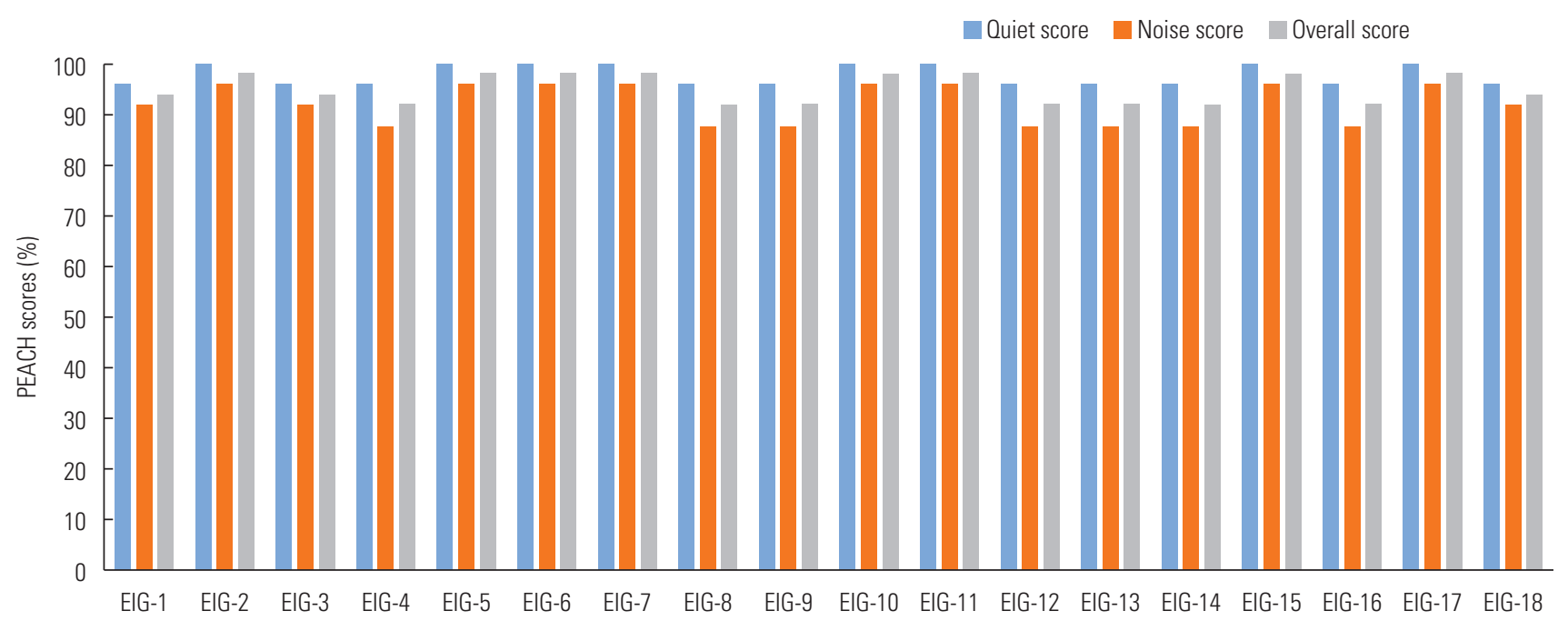

Figure 1. PEACH scores (\%) for quiet subscale, noise subscale, and overall score obtained by each participant of EIG.

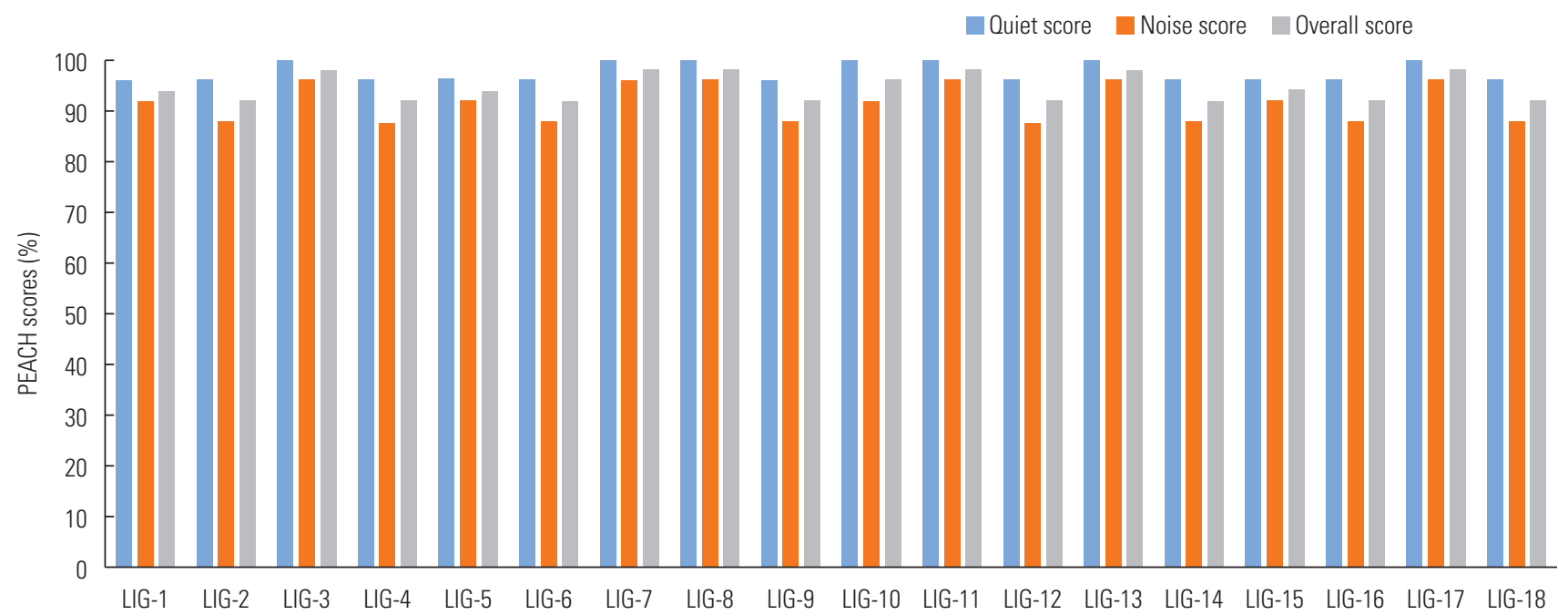

Figure 2. PEACH scores (\%) for quiet subscale, noise subscale, and overall score obtained by each participant of LIG. 
test reliability. Figures 1 and 2 show mean PEACH scores (\%) for quiet subscale, noise subscale, and combined subscale (overall score) of each participant of EIG and LIG. Figure 3 shows mean PEACH scores (\%) between participants of EIG and LIG for quiet subscale, noise subscale, and combined subscale (overall score). The results of the independent sam- ple t-test revealed that there was no statistically significant difference $(p>0.05)$ in auditory and communication skills in terms of mean PEACH scores (\%) between participants of EIG and LIG for quiet subscale score, noise subscale score, and overall score (Table 3 ).

Figure 4 shows mean PEACH scores (\%) among quiet sub-

Table 3. Independent sample t test results showing comparison between EIG and LIG for quiet subscale score, noise subscale score overall score

\begin{tabular}{|c|c|c|c|c|c|c|c|c|c|c|}
\hline \multirow{3}{*}{\multicolumn{2}{|c|}{$\begin{array}{l}\text { Comparison of mean PEACH scores } \\
\text { between EIG and LIG }\end{array}$}} & \multicolumn{2}{|c|}{ Equality of variance } & \multicolumn{7}{|c|}{ t-test of equality of means } \\
\hline & & \multirow{2}{*}{$\mathrm{F}$} & \multirow{2}{*}{ Sig } & \multirow{2}{*}{$\mathrm{t}$} & \multirow{2}{*}{$\mathrm{df}$} & \multirow{2}{*}{$\underset{\text { (2-tailed) }}{\text { Sig }}$} & \multirow{2}{*}{ MD } & \multirow{2}{*}{ SE } & \multicolumn{2}{|c|}{$95 \% \mathrm{Cl}$} \\
\hline & & & & & & & & & Lower & Upper \\
\hline \multirow[t]{2}{*}{ Quiet } & Equal Variances Assumed & 0.394 & 0.534 & 0.329 & 34 & 0.744 & 0.23166 & 0.7040 & -1.19908 & 1.66242 \\
\hline & Equal Variances not Assumed & & & 0.329 & 33.988 & 0.744 & 0.23166 & 0.7040 & -1.19910 & 1.66244 \\
\hline \multirow[t]{2}{*}{ Noise } & Equal Variances Assumed & 0.163 & 0.689 & 0.188 & 34 & 0.852 & 0.23111 & 1.2307 & -2.270113 & 2.73233 \\
\hline & Equal Variances not Assumed & & & 0.188 & 33.967 & 0.852 & 0.23111 & 1.2307 & -2.270202 & 2.73242 \\
\hline \multirow[t]{2}{*}{ Overall } & Equal Variances Assumed & 0.013 & 0.911 & 0.247 & 34 & 0.806 & 0.23138 & 0.9369 & -1.672721 & 2.13549 \\
\hline & Equal Variances not Assumed & & & 0.247 & 33.984 & 0.806 & 0.23138 & 0.9369 & -1.672753 & 2.13553 \\
\hline
\end{tabular}

MD: Mean Difference; SE: Standard Error; Cl: Confidence Interval.

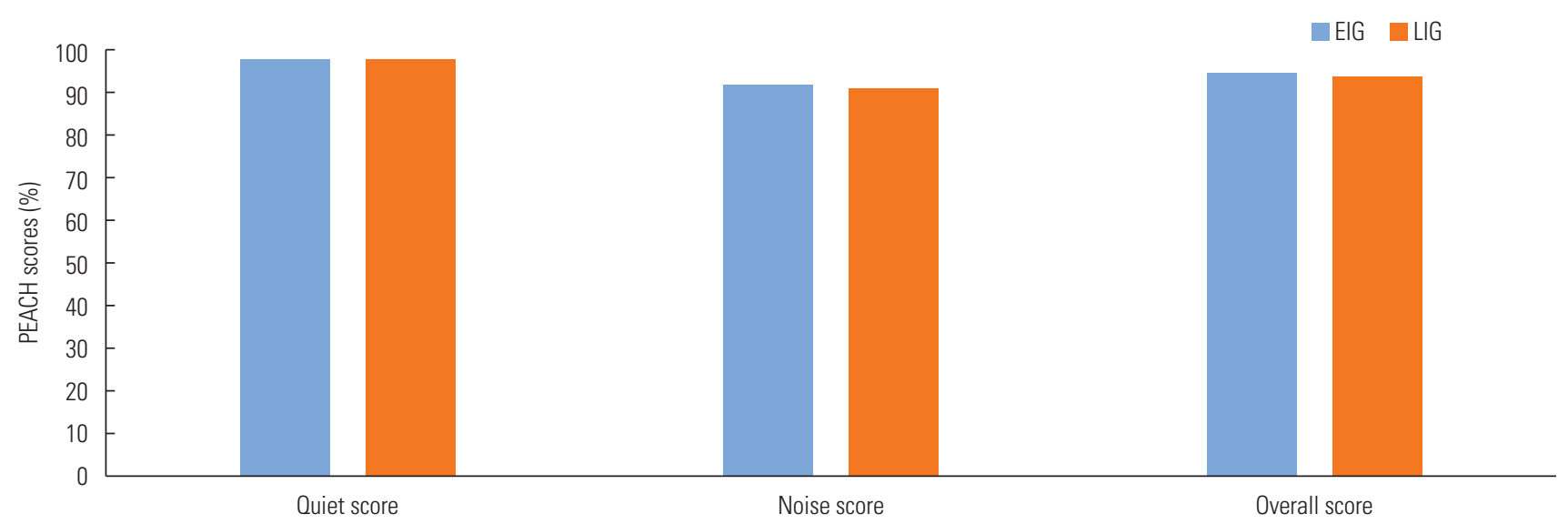

Figure 3. PEACH scores (\%) for quiet subscale, noise subscale, and overall score obtained by participants of EIG and LIG.

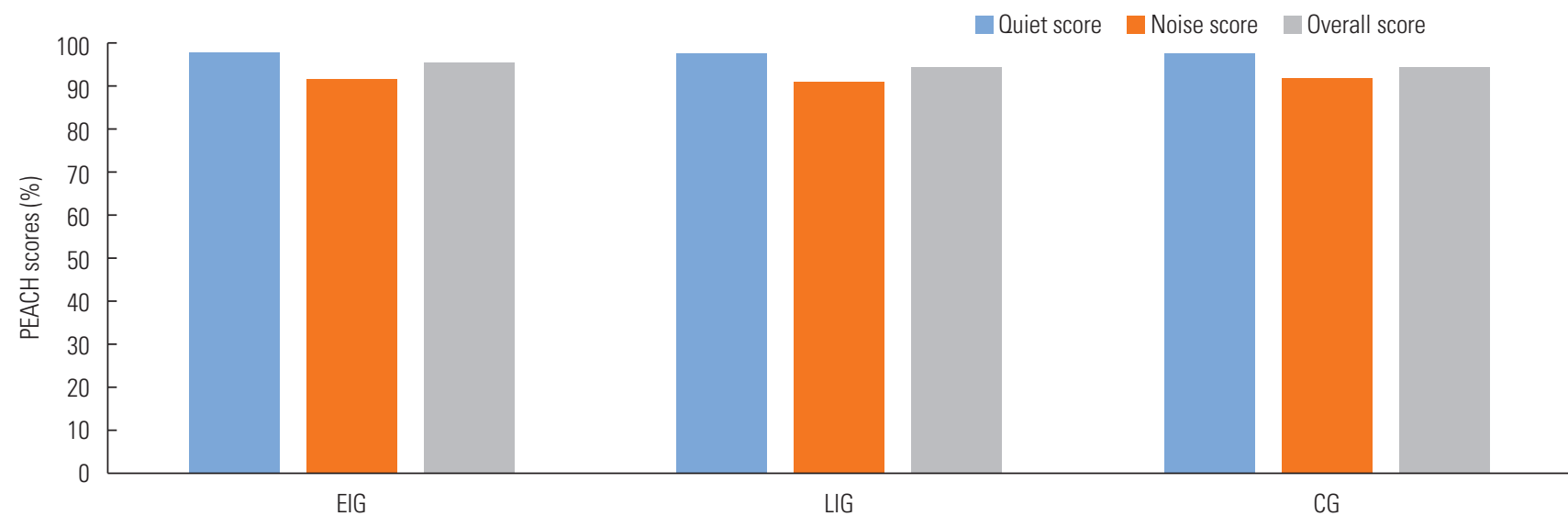

Figure 4. PEACH scores (\%) for quiet subscale, noise subscale score, and overall score obtained by participants of EIG, LIG, and CG. 
scale, noise subscale, and combined subscale (overall score) for participants of EIG and LIG. The results of one-way ANOVA revealed that there was a statistically significant difference $(p<0.05)$ between and within mean PEACH scores (\%) for participants of EIG, LIG, and CG. The participants of EIG, LIG, and CG obtained a lower mean PEACH score (\%) for noise subscale as compared to quiet subscale and overall score (Table 4). When the data were further subjected to LSD posthoc analysis, the results revealed that there was a statistically significant difference $(p<0.05)$ between quiet subscale score and noise subscale score, quiet subscale score and overall score, and noise subscale score and overall score (Table 5).

Table 4. One-way ANOVA results showing comparison between and within mean PEACH score (\%) among quiet subscale, noise subscale and overall score for children of EIG, LIG and CG

\begin{tabular}{llrrrrr}
\hline \multirow{2}{*}{ Group } & & $\begin{array}{c}\text { Sum of } \\
\text { Squares }\end{array}$ & df & $\begin{array}{c}\text { Mean } \\
\text { Square }\end{array}$ & F & Sig \\
\hline EIG & Between Groups & 351.688 & 2 & 175.844 & 20.700 & 0.000 \\
& Within Groups & 433.244 & 51 & 8.495 & & \\
& Total & 784.932 & 53 & & & \\
LIG & Between Groups & 351.625 & 2 & 175.813 & 19.900 & 0.000 \\
& Within Groups & 450.581 & 51 & 8.835 & & \\
& Total & 802.206 & 53 & & & \\
CG & Between Groups & 703.313 & 2 & 351.656 & 41.709 & 0.000 \\
& Within Groups & 885.271 & 105 & 8.431 & & \\
& Total & $1,588.584$ & 107 & & & \\
\hline
\end{tabular}

\section{DISCUSSION}

A systematic way of evaluating the outcomes of children with $\mathrm{CI}$ is an important aspect at every phase of post cochlear implantation rehabilitation. Considering the growing number of children with $\mathrm{CI}$ in southern states of India who are native speakers of Telugu, we have adapted and translated PEACH scale in Telugu, and administered on parents intending to assess and compare the auditory and communication skills of children as a function of the age at which they received CI. The results revealed no significant difference in auditory and communication skills in terms of mean PEACH scores between children of EIG and LIG for quiet subscale, noise subscale, and combined subscales (overall score). The findings of the present study suggest that the age at which the child received CI did not appear to be a major contributing factor in predicting the auditory and communication skills of children up to 5 years of age. A similar argument was made by Geers et al. [18] who have reported that children who received CI in the age range of 2 to 3 years of did not appear to achieve significantly greater communicative competence as compared to children who received $\mathrm{CI}$ in the age range of 4 to 5 years. Also, Moog \& Geers [22] reported from their study that children who achieved the best auditory skills were those who were implanted by 4.5 years of age, and children who achieved the best auditory skills developed language skills that corresponded or exceeded those of their normal-hearing age mates. Furthermore, it was reported that children with average learning ability who received CI at or before 5 years of age have greater potential to express and comprehend spoken

Table 5. LSD post-hoc multiple comparison of mean PEACH scores (\%) among quiet subscale, noise subscale and overall score for participants of EIG, LIG and CG

\begin{tabular}{|c|c|c|c|c|c|c|c|}
\hline \multirow{2}{*}{ Group } & \multicolumn{2}{|c|}{ Subscale } & \multirow{2}{*}{ MD (I-J) } & \multirow{2}{*}{ SE } & \multirow{2}{*}{ Sig } & \multicolumn{2}{|c|}{$95 \% \mathrm{Cl}$} \\
\hline & I & $\mathrm{J}$ & & & & Lower bond & Upper bond \\
\hline \multirow[t]{3}{*}{ EIG } & Quiet & Noise & $6.251111^{*}$ & 0.97153 & 0.000 & 4.30067 & 8.20156 \\
\hline & & Overall & $3.125556^{*}$ & 0.97153 & 0.002 & 1.17511 & 5.07600 \\
\hline & Noise & Overall & $-3.125556^{*}$ & 0.97153 & 0.002 & -5.07600 & -1.17511 \\
\hline \multirow[t]{3}{*}{ LIG } & Quiet & Noise & $6.250556^{*}$ & 0.99078 & 0.000 & 4.26147 & 8.23964 \\
\hline & & Overall & $3.125278^{*}$ & 0.99078 & 0.003 & 1.13619 & 5.11437 \\
\hline & Noise & Overall & $-3.125278^{*}$ & 0.99078 & 0.003 & -5.11437 & -1.13619 \\
\hline \multirow[t]{3}{*}{ CG } & Quiet & Noise & $6.250833^{*}$ & 0.68439 & 0.000 & 4.89380 & 7.60786 \\
\hline & & Overall & $3.125417^{*}$ & 0.68439 & 0.000 & 1.76839 & 4.48245 \\
\hline & Noise & Overall & $-3.125417^{*}$ & 0.68439 & 0.000 & -4.48245 & -1.76839 \\
\hline
\end{tabular}

The mean difference is significant at the 0.05 level.

MD: Mean Difference; SE: Standard Error; Cl: Confidence Interval. 
language at levels that are comparable with a similar-age peer group with normal hearing [18]. Hence, it can be inferred from the findings of the present study that children who receive CI up to the age of 5 years achieve similar auditory and communication skills irrespective of the age at which they receive CI.

While, several studies have reported that children who receive $\mathrm{CI}$ at a very younger age are expected to attain superior speech and language skills than children who receive $\mathrm{CI}$ at a later age $[10,16,17]$, there was no significant difference noticed in terms of auditory and communicative performance between children who received $\mathrm{CI}$ in the age range of 2 to 3 years (EIG) and children who received $\mathrm{CI}$ in the age range of 4 to 5 years (LIG) in the present study. Although the children of LIG received $\mathrm{CI}$ in the age range of 4 to 5 years, they had 2 to 3 years of pre-implant intervention with HAs, in addition to 5 to 6 years of post-implant CI use. The pre-implant intervention with HAs might provide children with advantages of early auditory stimulation and serve as a bridge to offer some amount of auditory access to speech and language until they receive CI $[23,24]$. There has been rapid and continuous progress in the CI technology in terms of the electrode array, speech coding strategies as well as surgical procedures to implant electrodes to improve auditory speech perception $[25,26]$. Hence, following implantation, the dramatic increase in access to auditory speech perception offered through modern CI devices might result in overall improvements in auditory development $[27,28]$. Further, with increased duration of CI use, children develop more access to auditory speech perception to gain greater improvements in auditory performance and develop receptive and expressive language skills across time $[14,15,20,29,30]$. Studies have also reported that children with $\mathrm{CI}$ achieved speech, language, and communication levels similar to their age-matched peers with normal hearing after up to 5 years of CI use $[18,20]$.

Studies have reported that intervention with an oral mode of communication method that emphasizes the use of speech and audition skills during communication provided a significant advantage for both spoken and total language skills [14, $18,20,31,32)$. In addition to pre-implant hearing with HAs and post-implant CI use, children of LIG in the present study have undergone 4 to 5 years of post-implant intervention in an oral mode of communication method, and are currently attending age-appropriate mainstream education with oral language as the main mode of communication. Hence, although, children of LIG would have displayed a gap in their auditory and com- municative skills compared to children of EIG at the time of implantation, the pre-implant HA usage [23,24], advances in CI technology [25,26], longer duration of post-implant CI usage $[20,30]$, intervention in an oral mode of communication [31,32] might have likely supported LIG to develop auditory and communication skills at par with EIG in the present study. Hence, there was no significant difference perceived in auditory and communicative performance between children of EIG and LIG in terms of mean PEACH scores for quiet subscale, noise subscale, and overall score in the present study.

Although the performance of children of EIG and LIG was similar in quiet and noisy communication environments, on average, both groups achieved significantly lower auditory and communicative performance in noisy communication environments as compared to quite communication environment. The poor representation of low-frequency pitch information [33] and limited spectral and temporal resolution [34] provided by implants might account for the poor performance in noisy communication environments. Providing binaural hearing is a vital component of aural rehabilitation as it helps to understand speech better in noisy listening environments and localize a sound source. However, most of the children who participated in the present study are limited to monaural CI. Hence, these children would not have availed the advantages of binaural hearing. Although binaural HA fitting has been a traditional practice while dealing with individuals with bilateral hearing impairment, monaural stimulation is commonly associated with cochlear implantation. This is especially common in the developing world like India due to financial barriers to opt for bilateral cochlear implantation [35]. Therefore, children with monaural CI are not only limited to the method of hearing (limitations of electrical stimulation provided by CI) but also with monaural hearing [13].

From the findings of the present study, it can be summarised that the translated and adapted PEACH scale can be considered a clinically feasible subjective tool for use in Telugu language in assessing the auditory and communicative performance of children with CI. Unlike many other subjective measures which require parents to just respond in terms of 'presence' or 'absence' of auditory behaviour of their children on checklists, the PEACH scale provides parents an opportunity to observe their children in everyday communication environments and note down as many responses of auditory and communicative behaviour of their children freely instead of restricting their responses to each item in the questionnaire. Additionally, there is flexibility in conducting 
another structured interview after completion of a questionnaire that gives an added opportunity for clinicians to review the responses, and also for parents to clarify their doubts on questions/responses in increasing the accuracy of response behaviour of their children. The measures of the PEACH scale can be valuable in monitoring the progress of children with CI at every stage of post-implant rehabilitation.

Besides, the findings of the present study highlight the significance of pre-implant HA usage, longer duration of postimplant CI usage, and oral mode of communication as an intervention method, in addition to the significance of advances in modern CI devices in achieving auditory and communicative performance among children who received CI in the later age. The findings of the present study furthermore, highlight the need for binaural hearing for children with CI to improve auditory and communicative performance in noisy communication environments. Hence, finance being the major concern in developing country like India for opting bilateral CI, a least-expensive and non-invasive method, i.e., bimodal stimulation can be an option for children who are limited to monaural CI to achieve some of the benefits of binaural hearing. However, we could not compare auditory and communicative performance of children with CI between monaural (CI alone) and bimodal (CI+HA) listening conditions due to limited number of children (EIG $=5$; LIG $=4$ ) with bimodal stimulation in the present study. Hence, highlights need for further study to assess the auditory and communication skills of children with $\mathrm{CI}$ as function of mode stimulation, i.e., monaural stimulation and bimodal stimulation using Telugu PEACH scale. Finally, the measures of the PEACH scale in combination with objective measures are worthwhile in obtaining more precise information on auditory and communicative performance, otherwise also in complimenting and validating the findings obtained from objective measures.

\section{CONCLUSIONS}

The findings of the present study revealed that the age at which the children received CI did not seem to be a major contributor in predicting the auditory and communicative performance. The variables such as pre-implant HA usage, longer duration of post-implant CI usage, oral mode of the post-implant intervention program, and oral mode of communication at educational setting, in addition to advances in CI technology, would add advantages for children who received $\mathrm{CI}$ in the later age. Despite superior performance in quiet communica- tion environments, the presence of background noise continued to degrade the auditory and communicative performance of children of both groups. Hence, highlights the need for bilateral and/or bimodal stimulation to achieve some of the benefits of binaural hearing. The translated and adapted PEACH scale can be considered a clinically feasible tool for use in Telugu language in obtaining comprehensive information on the functional auditory and communicative performance of children with CI. Further research needs to be carried out to establish norms for a larger sample of children with normal hearing and children using hearing devices such as HAs and CIs, and other assistive listening devices.

\section{REFERENCES}

1. Ching TYC, Dillon H, Marnane V, Hou S, Day J, Seeto M, et al. Outcomes of early- and late-identified children at 3 years of age: Findings from a prospective population-based study. Ear and Hearing. 2013;34:535-552.

2. Northern JL, Downs MP. Hearing in Children. 6th Ed, Baltimore: Williams \& Wilkins. 2014.

3. Yawn R, Hunter JB, Sweeney AD, Bennett ML. Cochlear implantation: A biomechanical prosthesis for hearing loss. F1000Prime Reports. 2015;7-45.

4. Zaidman-Zait A, Most T. Cochlear implants in children with hearing loss: Maternal expectations and impact on the family. Volta Review. 2005;105:129-150.

5. Bagatto MP, Moodie ST, Seewald RC, Bartlett DJ, Scollie SD. A Critical Review of Audiological Outcome Measures for Infants and Children. Trends in Amplification. 2011;15:23-33.

6. Quar TK, Ching TYC, Mukari SZ, Newall P. Parents' Evaluation of Aural/Oral Performance of Children (PEACH) Scale in the Malay Language: Data for Normal Hearing Children. International Journal of Audiology. 2012;51:326-333.

7. Boudreau D. Use of a Parent Questionnaire in Emergent and Early Literacy Assessment of Preschool Children. Language, Speech, Hearing Services in Schools. 2005;36:33-47.

8. Ching TYC, Hill M. The Parents' Evaluation of Aural/Oral Performance of Children (PEACH) Scale: Normative Data. Journal of American Academy of Audiology. 2007;18:220-235.

9. Lin FR, Wang NY, Fink NE, Quittner AL, Eisenberg LS, Tobey EA. CDaCI Investigative Team Assessing the use of Speech and Language Measures in Relation to Parental Perceptions of Development after Early Cochlear Implantation. Otology \& Neurotology. 2008;29:208-213.

10. Kumar S, Rout N, Kumar N, Chatterjee I, Selvakumaran H. Performance of Indian children with cochlear implant on PEACH scale. ISRN Otolaryngology. 2013;565096.

11. Waghulde P, Kumar SBR, Shora S, Kabani Z. Aural/Oral Performance of Children with Cochlear Implant under Monaural and Bimodal Listening Conditions: A Parental Evaluation. Communi- 
cation and Linguistics Studies. 2019;5:8-13.

12. Levinger $M$, Ronen $T$. Is it really clear? Adapting research tools for the needs of the deaf population. Journal of Social Work. 2008;8: 399-430.

13. Kumar SBR, Mohanty P. Bimodal Stimulation for Cochlear Implant Recipients: Speech Recognition Performance of Children with Cochlear Implant using Bimodal Stimulation. Deutschland: LAP LAMBERT Academic Publishing. 2016.

14. Kirk KI, Miyamoto RT, Lento CL, Ying E, O'Neill T, Fears B. Effects of age at implantation in young children. Annals of Otology, Rhinology, and Laryngology (Supplement). 2002;189:69-73.

15. Svirsky MA, Teoh S, Neuburger H. Development of language and speech perception in congenitally, profoundly deaf children as a function of age at implantation. Audiology \& Neurotology. 2004;9: 224-233.

16. Nicholas JG, Geers AE. Will they catch up? Journal of Speech Language and Hearing Research. 2007;50:1048-1062

17. Hayes H, Geers AE, Treiman R, Moog JS. Receptive vocabulary development in deaf children with cochlear implants: achievement in an intensive auditory-oral educational setting. Ear and Hear. 2009;30:128-35.

18. Geers AE, Nicholas JG, Sedey AL. Language Skills of Children with Early Cochlear Implantation. Ear and Hearing. 2003:24;46S-58S.

19. Geers AE. Speech, language, and reading skills after early cochlear implantation. Archives of Otolaryngology Head Neck Surgery. 2004;130:634-638.

20. Connor C, Craig H, Raudenbush S, Heavner K, Zwolan T. The age at which young deaf children receive cochlear implants and their vocabulary and speech-production growth: Is there an added value for early implantation? Ear and Hearing. 2006;27:628-644.

21. James D, Rajput K, Brinton J, Goswami S. Phonological Awareness, Vocabulary, and Word Reading in Children Who Use Cochlear Implants: Does Age of Implantation Explain Individual Variability in Performance Outcomes and Growth? Journal of Deaf Studies and Deaf Education. 2008;13:117-137.

22. Moog J, Geers A. Speech and language acquisition in young children after cochlear implantation. The Otolaryngology Clinics of North America. 1999;32:1127-1142.

23. Nicholas JG, Geers AE. Effects of early auditory experience on the spoken language of deaf children at 3 years of age. Ear and Hearing. 2016;27:286-298.

24. Rinella H. Cochlear Implants: Factors Influencing Speech and Language Development in Children, Carbondale: Southern Illi- nois University Carbondale. 2014.

25. Gifford RH, Olund AP, Dejong M. Improving speech perception in noise for children with cochlear implants. Journal of American Academy of Audiology. 2011;22:623-632.

26. Sampaio AL, Araujo MF, Oliveira CA. New criteria of indication and selection of patients to cochlear implant. International Journal of Otolaryngology. 2011;1-13.

27. Krueger B, Joseph G, Rost U, Strauss-Schier A, Lenarz T, Buechner A. Performance groups in adult cochlear implant users: speech perception results from 1984 until today. Otology \& Neurotology. 2008;29:509-512.

28. Huttunen K, Rimmanen S, Vikman S, Virokannas N, Sorri M, Archbold S, Lutman ME. Parents' views on the quality of life of their children 2-3 years after cochlear implantation. International Journal of Paediatric Otorhinolaryngology. 2009;73:1786-94.

29. Miyamoto RT, Kirk KI, Svirsky M, Sehgal S. Longitudinal communication skill acquisition in paediatric cochlear implant recipients. Advances in Otorhinolaryngology. 2000;57:212-214.

30. Dettman SJ, Pinder D, Briggs RJS, Dowell RC, Leigh JR. Communication development in children who receive the cochlear implant younger than 12 months: risks versus benefits. Ear and Hearing. 2007;28:11S-18S.

31. Marschark M, Spencer PE. Spoken language development of deaf and hard-of-hearing children: Historical and theoretical perspectives. In P. E. Spencer \& M. Marschark (Eds.), Advances in the spoken language development of deaf and hard-of-hearing children (pp. 3-21). New York: Oxford University Press. 2006.

32. Holt RF, Svirsky MS. An exploratory look at paediatric cochlear implantation: is earliest always best? Ear and Hearing. 2008;29: 492-511.

33. Cullington HE, Zeng FG. Bimodal Hearing Benefit for Speech Recognition with Competing Voice in Cochlear Implant Subject with Normal Hearing in Contralateral Ear. Ear and Hearing. 2010;31:70-73.

34. Yuksel M, Meredith MA, Rubinstein JT. Effects of Low Frequency Residual Hearing on Music Perception and Psychoacoustic Abilities in Paediatric Cochlear Implant Recipients. Frontiers in Neuroscience. 2019;13:1-11.

35. Kumar SBR, Mohanty P, Prakash SGR. Speech Recognition Performance in Children with Cochlear Implant using Bimodal Stimulation, Indian Journal of Otolaryngology and Head Neck Surgery. 2010;62:342-345. 